\title{
Intensidade de esforço realizado durante partidas amistosas de Futebol 7
}

http://dx.doi.org/10.11606/1807-5509201700040767

\author{
Reinaldo BINATTI NETO* \\ Renan Felipe Hartmann NUNES* \\ Francimara Budal ARINS * \\ Tiago Martins COELHO* \\ Naiandra DITTRICH* \\ Priscila Cristina dos SANTOS* \\ Ricardo Dantas de LUCAS* \\ Luiz Guilherme Antonacci GUGLIELMO* \\ *Centro de Desportos, \\ Universidade Federal \\ de Santa Catarina, \\ Florianópolis, SC, \\ Brasil.
}

\section{Resumo}

0 objetivo deste estudo foi identificar a intensidade de esforço requerida durante 4 partidas amistosas e a distância percorrida em cada um dos domínios fisiológicos (moderado, pesado e severo). Dez jogadores adultos $(25,2 \pm 3,7$ anos; $72,0 \pm 8,9 \mathrm{~kg} ; 175,3 \pm 6,9 \mathrm{~cm} ; 12,4 \% \pm 3,1$ gordura) de uma equipe amadora de Futebol 7 participaram do estudo. Os participantes foram submetidos a cinco sessões experimentais com um intervalo de 48 horas para cada sessão. Na primeira sessão experimental foram realizadas medidas antropométricas seguidas de um teste incremental intermitente de campo (T-CAR) para a avaliação da frequência cardíaca máxima $\left(\mathrm{FC}_{\text {Máx }}\right)$, pico de velocidade $(\mathrm{PV})$ e identificação dos domínios fisiológicos a partir do ponto de deflexão da frequência cardíaca. Nas sessões seguintes, a frequência cardíaca (FC) e o perfil das atividades realizadas pelos jogadores (Sistema Global de Posicionamento - GPS) ou (global positioning system - GPS) foram mensurados durante quatro partidas amistosas. Para a análise estatística utilizou-se a ANOVA two way para medidas repetidas combinado com o teste de Bonferroni. Não foram encontradas diferenças estatisticamente significativas nos valores médios da FC entre os jogos e entre $01^{\circ}$ tempo e $2^{\circ}$ tempo. Em relação à distância percorrida, os resultados encontrados mostraram que os atletas permaneceram maior parte do tempo se exercitando no domínio moderado $(664,2 \pm 62$ $m ; 45,5 \%)$ quando comparado com o domínio pesado $(437 \pm 45 \mathrm{~m}$ m; 31,5\%) e severo $(284,3 \pm 66 \mathrm{~m}$; $23,5 \%)(p<0,05)$. Entretanto, quando somados os valores de permanência nos domínios pesado e severo, podemos observar que mais de 50\% do tempo total de jogo foram realizados em altas intensidades. Assim, pode-se concluir que o Futebol 7 é um esporte de alta demanda fisiológica.

Palavras-chaves: Esportes Coletivos; Intensidade do Exercício; Frequência Cardíaca; Desempenho.

\section{Introdução}

O Futebol 7 (F7) é um esporte coletivo jogado entre dois times de 7 jogadores cada um com duraçáo de dois tempos de 25 minutos e com intervalo de 10 minutos entre os 2 tempos. A história desta modalidade ainda não é bem clara, mas sabe-se que é um esporte originado do futebol, com adaptações nas regras e dimensóes do campo. O esporte é conhecido por diferentes nomes (futebol society, futebol social, futebol suíço), mas é oficialmente registrado e oficializado pelo Conselho Nacional de Esportes, como Futebol $7^{1}$.
O F7 é um esporte caracterizado pela combinação de açôes de elevadas intensidades, intercalados com períodos de recuperação, e sob o ponto de vista fisiológico, uma modalidade que depende tanto de variáveis relacionadas ao metabolismo aeróbio quanto anaeróbio, semelhante a modalidades como o futsal e o futebol $^{2,3}$. Porém, apesar de sua popularidade, principalmente no Brasil, poucos são os estudos que analisaram as demandas fisiológicas em atletas de $F 7^{4,5}$. 
A identificação das exigências físicas impostas aos jogadores durante as partidas são fundamentais para a formulação de um programa de treinamento adaptado às necessidades específicas do esporte ${ }^{3,4}$. Desta maneira, identificar as demandas do jogo e controlar as cargas de treino (interna e externa) tornase fundamentais para o entendimento das demandas físicas e consequente melhora no desempenho esportivo $^{6,7}$.

Assim, a frequência cardíaca (FC) tem sido utilizada para quantificar a carga interna durante treinos e jogos ${ }^{3,4,8}$. Barbero-Álvarez et al. ${ }^{3}$, por exemplo, utilizaram valores médios da FC para quantificar a carga de um jogo de futsal. Além disso, demonstraram que a distância total percorrida (\%) em velocidade máxima durante a partida foi realizada acima de $85 \%$ da frequência cardíaca máxima $\left(\mathrm{FC}_{\text {Máx }}\right)$ por mais de $80 \%$ do tempo em quadra. Enquanto, para atletas de futebol, a intensidade de jogo pode variar entre $80-90 \% \mathrm{FC}_{\text {Máx }}{ }^{9}$ e de $\mathrm{F} 7$ atingem valores acima de $80 \%$ da $\mathrm{FC}_{\text {Máx }}$ por mais de $80 \%$ do tempo total de jogo 5 . Além de ser um método não invasivo e de fácil utilização, a FC também pode ser utilizada durante testes (Teste de Carminatti T-CAR) ${ }^{10,11}$ como um instrumento fidedigno para determinar os domínios de transição fisiológica (moderado, pesado e severo) de exercício por meio do ponto de deflexão da frequência cardíaca $(\mathrm{PDFC})^{12}$. Estudos têm demonstrado que o monitoramento do pico de velocidade (PV) e do segundo limiar de transição fisiológico (LTF2), no qual corresponde a 80,4\% do $\mathrm{PV}$, obtidos durante o T-CAR ${ }^{10,13}$, estão associados

\section{Método}

\section{Delineamento experimental}

Este estudo pode ser caracterizado quanto a sua natureza como aplicada, quanto à abordagem do problema como quantitativo de caráter transversal e quanto aos objetivos como descritiva ${ }^{15}$. A seleçáo dos atletas foi não probabilística, do tipo intencional, tendo como critério para seleção os jogadores pertencentes a uma equipe que disputa campeonatos oficiais da modalidade na cidade de Florianópolis/SC.

Este projeto foi aprovado pelo Comitê de Ética em Pesquisa com Seres Humanos (CEPSH) da Universidade Federal de Santa Catarina (UFSC) com registro no CAAE número: 33228214.0.0000.0121 e com medidas-padrão realizadas em laboratório, no qual permitem verificar a intensidade de esforço durante jogos e treinos.

O Global position system (GPS) também tem sido utilizado em diversas modalidades como ferramenta para mensurar a distância total percorrida em diferentes intensidades ${ }^{4,5,14}$. Este equipamento permite identificar com maior precisão as demandas fisiológicas dos atletas durante jogos e treinos, nível de rendimento durante uma temporada, conhecer as diferenças destas demandas entre determinadas posiçóes, e desta maneira, auxiliar no aperfeiçoando do processo de treinamento. GómEZ et al. ${ }^{5}$, encontraram diferenças significativas entre a distância percorrida em atletas amadores de F7 em diferentes intensidade e posiçóes. Além disso, os autores reportam que este equipamento pode ser eficaz para quantificar as demandas neuromusculares, cardiovasculares e metabólicas durante as partidas.

A literatura apresenta diversos estudos acerca de modalidades como o futsal e o futebol, entretanto, apesar da semelhança dessas modalidades com o F7, sabe-se que fatores como número de jogadores, dimensão do campo, tempo de jogo e regras alteram as características do jogo e consequentemente as demandas fisiológicas impostas. Dessa forma, considerando que poucos são os estudos que analisaram as demandas fisiológicas em atletas durante partidas de $\mathrm{F}^{4,5}$, o objetivo deste estudo foi identificar a intensidade de esforço requerida durante 4 partidas amistosas e a distância percorrida em cada um dos domínios fisiológicos.

parecer de aprovação número: 056891/2014. Antes de iniciarem os procedimentos para a coleta de dados, os atletas participantes do estudo foram esclarecidos sobre os objetivos e os métodos da pesquisa e na sequência assinaram o Termo de Consentimento Livre e Esclarecido (TCLE).

\section{Participantes}

A amostra da pesquisa foi composta por 10 jogadores adultos (idade: $25,2 \pm 3,7$ anos; massa corporal: 72,0 $\pm 8,9 \mathrm{~kg}$; estatura: $175,3 \pm 6,9 \mathrm{~cm}$; percentual de gordura corporal: $12,4 \% \pm 3,1$ ) de uma equipe amadora de F7. 
As coletas dos dados foram realizadas no período competitivo, sendo que, nesta fase os atletas treinavam sistematicamente duas vezes por semana (60-90min por sessão), no período noturno, além de jogos amistosos/oficiais no final de semana. Os treinamentos eram separados principalmente para melhora do desempenho técnico/tático e físico (especificamente dos sistemas anaeróbios e aeróbios). Os treinamentos anaeróbios consistiam de exercícios pliométricos, de força explosiva e de sprints repetidos, enquanto que para o treinamento aeróbio os atletas realizavam corridas com diferentes intervalos de duração. Além disso, os atletas também realizavam atividades para melhorar a capacidade aeróbia/anaeróbia por meio da execução de jogos reduzidos (JRs). Pois, dependendo da estrutura de treino dos JRs sobre os parâmetros que compóem o jogo, como o espaço (dimensão), tempo, tática, número de atletas, entre outros, influenciam as respostas físicas e fisiológicas, potencializando mais o componente aeróbio ou anaeróbio, dependendo do objetivo do treinamento.

\section{Procedimento}

Os participantes foram submetidos a cinco sessóes experimentais com um intervalo exato de 48 horas para cada sessão. Na primeira sessão foi realizada uma avaliação antropométrica para caracterizaçáo da amostra seguido de um teste incremental intermitente de campo (T-CAR). $\mathrm{Na}$ segunda até a quinta sessão ocorreu o monitoramento das atividades realizadas durante quatro partidas amistosas (2 tempos de 25 minutos com intervalo de 10 minutos entre os tempos) com diferentes equipes intercaladas exatamente por 48 horas de descanso. Somente seis jogadores iniciavam as partidas, sendo que durante as mesmas ocorreram substituiçôes com o passar do tempo. O critério de substituição do atleta em campo foi determinado em relação ao seu rendimento (técnico, tático e físico) durante a partida, porém, nenhum atleta permaneceu menos de $25 \%$ (15 minutos) do tempo total de jogo em cada partida. Buscando evitar quaisquer variaçōes circadianas intraindividuais todas as avaliaçóes foram realizadas em um mesmo horário (19h). Para evitar desidratação, os atletas foram permitidos o consumo de água ad libitum. Todos os participantes foram instruídos a não realizar exercício físico no dia anterior, como também a não ingerir alimentos com alto teor energético e/ou bebida contendo cafeína por um período anterior a três horas do início das sessões.

Os procedimentos utilizados para realizar as mensuraçôes antropométricas seguiram os protocolos definidos em BenedetTi et al. ${ }^{16}$ A massa corporal foi medida utilizando-se uma balança com resoluçáo de $0,1 \mathrm{~kg}\left(\mathrm{TOLEDO}^{\circ}\right)$. Para a determinação da estatura foi utilizado um estadiômetro com resolução de $1 \mathrm{~mm}$ $\left(\mathrm{SANNY}^{\circledast}\right)$. Foram medidas três dobras cutâneas, com o adipômetro científico com resolução de $1 \mathrm{~mm}\left(\mathrm{CESCORF}^{\odot}\right)$. A densidade corporal (DC) foi estimada pela equação (1) de JACKsON e PoLLOCK ${ }^{17}$, com aplicação deste valor para estimar o percentual de gordura deste por meio da equação (2) de SIRI ${ }^{18}$.

$\mathrm{DC}=1,109380-0,0008627$ (peitoral + abdominal + coxa média $)+0,0000016$ (peitoral + abdominal + coxa média) ${ }^{2}-0,0002574 *$ (idade) (1)

$$
\% \mathrm{GC}=\% \text { (495/densidade corporal)-450 (2) }
$$

O Teste de Carminatti (T-CAR) é um teste com multiestágios de 90 segundos de duração em sistema “ida-e-volta”, sendo que cada estágio é constituído por 5 repetiçóes de 12 segundos de corrida (distância variável), intercaladas por 6 segundos de caminhada. O ritmo é ditado por um sinal sonoro (bip), em intervalos regulares de 6 segundos, na qual determina a velocidade de corrida a ser desenvolvida nos deslocamentos entre as linhas paralelas demarcadas no solo e também sinalizadas por cones. A velocidade inicial do teste é de $9,0 \mathrm{~km} \cdot \mathrm{h}^{-1}$ (distância inicial de $15 \mathrm{~m}$ ) com incrementos de $0,6 \mathrm{~km} \cdot \mathrm{h}^{-1}$ a cada estágio, até a exaustão voluntária, mediante aumentos sucessivos de $1 \mathrm{~m}$ a partir da distância inicial ${ }^{10}$. Durante o teste cada atleta utilizou um cardiofrequencímetro marca Polar (Electro-modelo S610), armazenadas em intervalos de 5 segundos. $\mathrm{O}$ teste foi considerado máximo sempre que o sujeito atingiu pelo menos $90 \% \mathrm{FC}_{\text {Máx }}$ predita $\left(\mathrm{FC}_{\text {Máxpred }}\right)$ conforme cálculo prévio através da fórmula 208 (0,7x idade) proposta por TANAKA et al. ${ }^{19} \mathrm{~A}$ maior velocidade alcançada no teste foi denominada de PV. Quando o sujeito interrompeu o teste antes de completar o estágio, o PV foi corrigido a partir da seguinte equação:

$$
\mathrm{PV}(\mathrm{km} \cdot \mathrm{h})=\mathrm{v}+[(\mathrm{nv} / 10) \cdot 0,6]
$$

Onde "v" é a velocidade do último estágio completo em km.h, o "nv" é o número de voltas percorridas no estágio incompleto, "10" é o número total de voltas de um estágio, excluindo as 4 voltas anunciadas na locuçáo do protocolo durante as 
pausas de 6 segundos de cada estágio e " 0,6 " é o incremento da velocidade.

O PDFC foi determinado por meio do método matemático DMAX proposto por KARA et al. ${ }^{20}$, plotando-se a resposta da FC (eixo y) obtida no final de cada estágio e a velocidade do protocolo TCAR (eixo $\mathrm{x}$ ), respectivamente. Os pontos foram ajustados por uma função polinomial de terceira ordem e, posteriormente, foi empregado um ajuste linear, utilizando-se os dois extremos da curva, derivando-se uma reta. O valor do DMAX foi definido como o ponto mais distante entre as duas linhas.

Para determinar a intensidade de esforço dos atletas durante as partidas amistosas foram delimitados domínios fisiológicos, referentes aos percentuais dos valores do PDFC, de acordo com o proposto por Almeida et al. ${ }^{11} \mathrm{O}$ domínio moderado foi delimitado por valores de $\mathrm{FC}$ que permaneceria abaixo de $80 \%$ do PDFC. O domínio pesado abrange os valores de FC entre $80 \%$ e $100 \%$ do PDFC e por fim, o domínio severo compreende valores de FC acima do PDFC.

A distância total percorrida em cada um dos domínios e a FC mensurada a cada cinco segundos foi analisado por meio de frequencímetros incorporados a Geografic Position System - GPS (KSPORTS ${ }^{\circledR}$ ) durante as 4 partidas amistosas que seguiram as mesmas regras de partidas oficiais. Para

\section{Resultados}

As variáveis referentes à $\mathrm{FC}$ e $\mathrm{PV}$ obtidas por meio do teste incremental de corrida intermitente TCAR para a identificação dos domínios fisiológicos estão apresentadas na TABELA 1. a quantificação da carga interna relativa à $\mathrm{FC}$ foi assumida a média da FC relativa (\%FCmax).

Para verificar a normalidade dos dados foi utilizado o teste de Shapiro-Wilk. O teste de Levene foi utilizado para testar a homecedasticidade, ao passo que a esfericidade dos dados foi verificada mediante o teste de Mauchly. Quando esse último pressuposto foi violado, a correção de GreenhouseGeisser foi adotada. Em razão da não violação paramétrica, utilizaram-se medidas de tendência central (média) e dispersão (desvio padrão) para descrever as variáveis da investigação. Uma Anova Two-way de mediadas repetidas foi utilizada para comparar as diferenças das variáveis $\mathrm{FC}$ em função dos jogos (J1, J2, J3, J4) e do tempo de jogo $\left(1^{\circ}\right.$ e $2^{\circ}$ tempo). A mesma análise foi utilizada para comparar os domínios fisiológicos (DM, DP, DS) entre o tempo de jogo ( $1^{\circ}$ e $2^{\circ}$ tempo). O teste post hoc de Bonferroni foi aplicado para identificar possíveis diferenças estatísticas. Ademais, calculouse o tamanho do efeito (TE) de $\operatorname{CoHeN}^{21}$ (d), para indicar diferenças do ponto de vista prático baseando-se nos seguintes critérios: $<0,35$ efeito trivial, 0,35-0,80 pequeno efeito, 0,80-1,50 grande efeito. Em todas as análises, realizadas no Statistical Package for Social Sciences (SPSS), versão 19.0 para Windows, foi adotado o valor de $\mathrm{p}<0,05$ para significância.
$\mathrm{FC}_{\text {Máx }}$ : Frequência cardíaca máxima; PV: Pico de velocidade; VPDFC: Ponte visual de deflexão da

frequência cardíaca; $\% \mathrm{FC}_{\text {Mäx }}$ : Percentual da frequência cardíaca máxima; VPDFC: Velocidade do Ponte deflexão da frequência cardíaca; $\%$ PV: Percentual do pico de velocidade; DP: Desvio padrão.
TABELA 1 - Valores relativos as demandas fisiológicas obtidas por meio do teste incremental de corrida intermitente.

\begin{tabular}{|c|c|c|c|}
\hline Variáveis $(n=10)$ & Média $\pm \mathrm{DP}$ & Mínimo & Máximo \\
\hline $\mathrm{FC}_{\text {Máx }}(\mathrm{bpm})$ & $196 \pm 7,0$ & 186 & 204 \\
\hline $\operatorname{PV}\left(\mathrm{km} \cdot \mathrm{h}^{-1}\right)$ & $15,8 \pm 0,8$ & 14,5 & 16,8 \\
\hline PDFC (bpm) & $179 \pm 6,0$ & 165 & 188 \\
\hline $\operatorname{PDFC}\left(\% \mathrm{FC}_{\text {Max }}\right)$ & $91,6 \pm 2,3$ & 88,7 & 95,3 \\
\hline $\operatorname{VPDFC}\left(\mathrm{km} \cdot \mathrm{h}^{-1}\right)$ & $12,9 \pm 0,3$ & 12,6 & 13,2 \\
\hline VPDF (\%PV) & $82,0 \pm 2,6$ & 78,6 & 86,9 \\
\hline
\end{tabular}


TABELA 2 - Valores relativos as demandas fisiológicas dos jogadores de F7 registrados durante as quatro partidas amistosas.

\begin{tabular}{ccccc}
\hline Partida & FC $(\mathbf{b p m})$ & $\mathbf{F C}_{\text {MÁxjoGo }} \mathbf{( b p m )}$ & $\mathbf{\% F C}_{\text {MÁxjoGo }}$ & \%FC $_{\text {Máx }}$ T-CAR \\
\hline J1 & $163 \pm 5$ & $193 \pm 6$ & $83,4 \pm 3,4$ & $83,1 \pm 4,8$ \\
\hline J2 & $162 \pm 10$ & $195 \pm 4$ & $83,1 \pm 4,3$ & $82,6 \pm 9,5$ \\
\hline J3 & $163 \pm 13$ & $192 \pm 5$ & $84,8 \pm 5,3$ & $83,1 \pm 13,2$ \\
\hline J4 & $162 \pm 6$ & $190 \pm 2$ & $85,2 \pm 2,8$ & $82,6 \pm 5,8$ \\
\hline J & $163 \pm 10$ & $192 \pm 3$ & $84,3 \pm 3,9$ & $83,0 \pm 8,3$ \\
\hline
\end{tabular}

Na FIGURA 1 estão apresentados os valores médios de FC dos jogadores de F7 registrados durante o $1^{\circ}$ e o $2^{\circ}$ tempo da partida durante a realizaçáo dos 4 jogos analisados. Não foram encontradas diferenças significativas na média da FC em funçáo dos jogos (TABELA 2) e entre $1^{\circ}$ e $2^{\circ}$ tempo.

As médias das distâncias percorridas durante os 4 jogos amistosos e entre o $1^{\circ}$ tempo e $2^{\circ}$ tempo em cada domínio fisiológico de intensidade (moderado, pesado e severo) estão apresentadas na FIGURA 2. Foram encontradas diferenças significativas nas médias da distância percorrida entre o domínio moderado comparado ao pesado $(664,2 \pm 62 \mathrm{~m}$; $437 \pm 45 \mathrm{~m} \mathrm{~F}=11,370 ; \mathrm{p}<0,01 ; \mathrm{TE}=4,19)$, e moderado ao severo $(664,2 \pm 62 \mathrm{~m} ; 284,3 \pm 66 \mathrm{~m}$; $\mathrm{F}=11,370 ; \mathrm{p}<0,01 ; \mathrm{TE}=5,93)$, porém não foi encontrada diferença significativa entre os tempos das partidas.

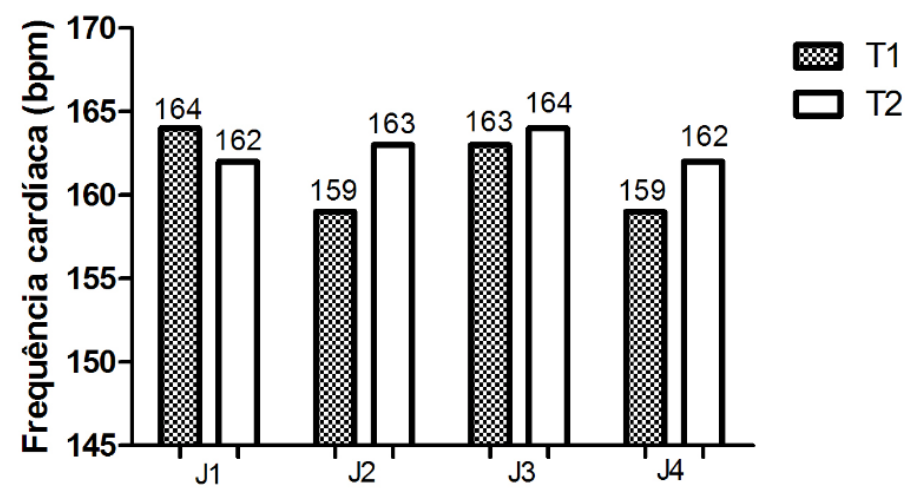

FIGURA 1 - Valores da média da FC dos jogadores de F7 registrados durante as quatro partidas e entre o $1^{\circ}$ e $2^{\mathrm{o}}$ tempo.

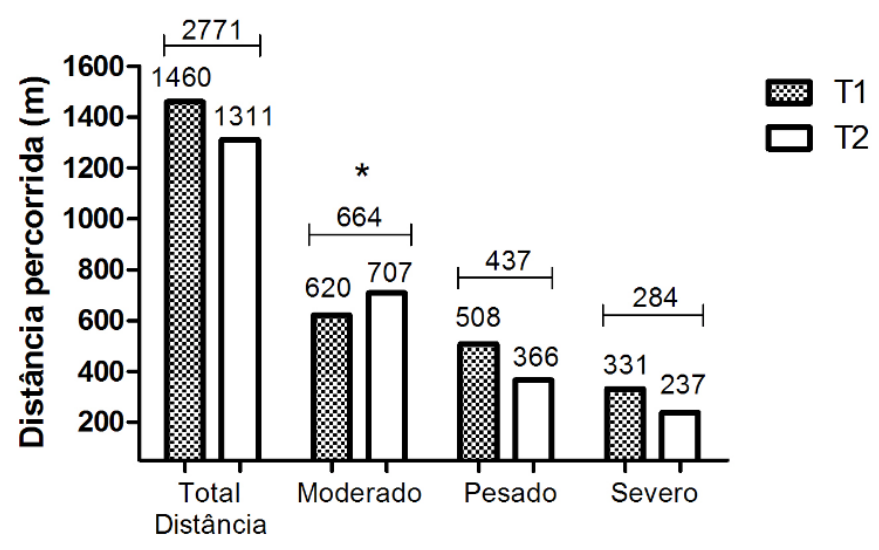

T1: primeiro tempo de partida; T2: segundo tempo de partida; " $p<0,05$ significativamente diferentes em relação ao domínio moderado para o domínio pesado e severo.

J: jogo; T1: primeiro tempo de partida; 72 : segundo tempo de partida.

\section{.}


T1: primeiro tempo de partida; T2: segundo tempo de partida; $p<0,05$ significativamente diferentes em relação ao domínio moderado para o domínio pesado e severo.
Os valores do tempo de permanência dos jogadores de F7 durante os 4 jogos amistosos e entre o $1^{\circ}$ tempo e $2^{\circ} \mathrm{em}$ cada domínio fisiológico (moderado, pesado, severo) estão apresentados na FIGURA 3. É possível observar que os mesmos permanecem a maior parte do tempo das partidas realizando açóes em intensidades inferiores ao LTF2, independente do período de jogo ( $1^{\circ}$ e $2^{\circ}$ tempo).
Nota-se que os atletas permanecem a maior parte do tempo no domínio moderado, apresentando diferença estatisticamente significativa em relação ao tempo de permanência no domínio pesado $(45,5 \% ; 31,5 \% ; \mathrm{F}=8,625 ; \mathrm{TE}=1,39)$ e severo $(45,5 \% ; 23,5 \% ; \mathrm{F}=8,625 \mathrm{TE}=2,38)$. Não foi encontrada diferença significativa entre os tempos das partidas.

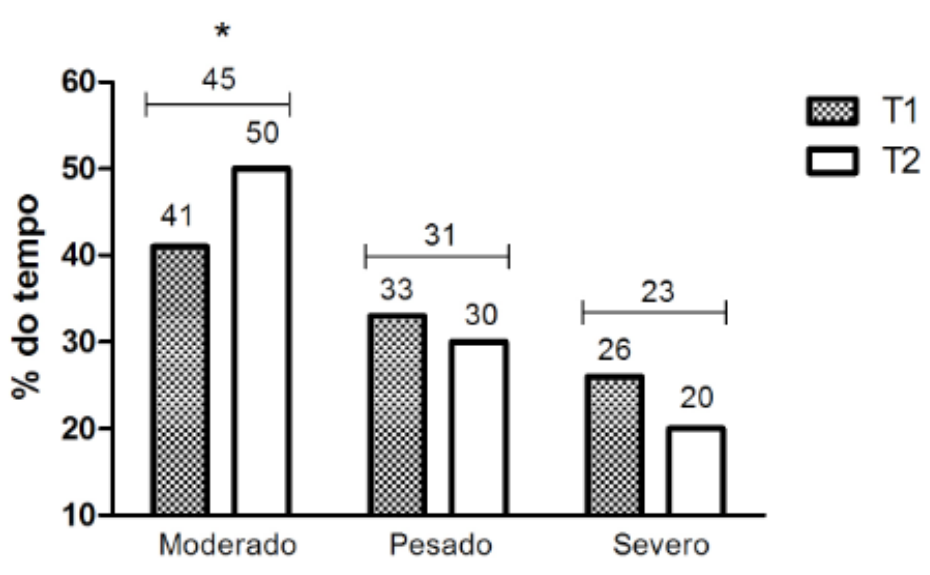

Domínios de intensidade

FIGURA 3 - Percentual do tempo total gasto nos 4 jogos em cada domínio fisiológico e entre o $1^{\mathrm{o}}$ e $2^{\mathrm{o}}$ tempo.

\section{Discussão}

O principal objetivo deste estudo foi analisar a intensidade de esforço dos jogadores de F7 durante 4 partidas amistosas. Os resultados mostram que os atletas permaneceram grande tempo das partidas se exercitando no domínio moderado, ou seja, em intensidades inferiores ao segundo limiar de transição fisiológica $\left(\mathrm{LTF}_{2}\right)$.

Em relação aos dados de FC média registrados nas partidas analisadas $\left(\mathrm{FC}_{\text {MEDJOGO }}\right.$ ), pode-se observar que a média foi similar entre os 4 jogos $(163 \pm 10 \mathrm{bpm})$, representando uma intensidade relativa de $84,3 \pm 3,9 \%$ da FC $_{\text {MÁXJOGO }}$ (TABELA 2). Estudos anteriores também avaliaram a FC média sustentada em partidas oficiais de futebol ${ }^{8} \mathrm{e}$ futsal ${ }^{3}$. Entretanto, ao contrário dos achados do presente estudo, os autores encontraram valores superiores de FC nas partidas de futebol e futsal $(170 \pm 9 \mathrm{bpm}$; $89 \%$ da $\mathrm{FC}_{\text {MÁXJOGO, }} 174 \pm 9 \mathrm{bpm} ; 90 \%$ da $\mathrm{FC}_{\text {MÁxJOGO }}$ respectivamente). Porém, é importante ressaltar que em ambos os estudos a amostra foi composta por atletas profissionais, diferente do presente estudo no qual participaram atletas considerados amadores. Dentre os poucos estudos que analisaram a demanda de jogo em atletas de F7, GómEz et al. ${ }^{5}$, ao analisarem 7 partidas, encontraram valores médios da FC de $167 \pm 10 \mathrm{bpm}\left(87 \pm 5 \%\right.$ das $\left.\mathrm{FC}_{\text {MÁxJOGO }}\right)$ com valores picos de $188 \pm 12 \mathrm{bpm}\left(97 \pm 6 \% \mathrm{FC}_{\mathrm{MAX}}\right)$, semelhante aos achados deste trabalho. Por outro lado, BarberoÁlvarez et al. ${ }^{4}$, reportaram resultados de $86 \%$ da $\mathrm{FC}_{\text {MÁXJOGO }}$ em atletas sub 12 e 14 , sugerindo que este formato de jogo é bastante exigente a partir de um ponto de vista cardiovascular.

Os resultados do presente estudo não reportaram diferença significativa da média da FC entre os jogos e entre o $1^{\circ}$ e $2^{\circ}$ tempo demonstrando assim que os atletas sustentaram a intensidade relativa entre os períodos, o que possivelmente se deve ao fato do F7 permitir substituiçóes ilimitadas. Porém, esses achados divergem de outros trabalhos realizados em modalidades como o futsal ${ }^{3}$, futebol ${ }^{22}$ e $\mathrm{F}^{4,5}$, no qual reportaram diferença entre os tempos. Segundo os mesmos autores esta diferença pode ser explicada pelo aumento dos níveis de fadiga causado pela elevada demanda de jogo, reduzindo assim a capacidade do músculo para gerar força ${ }^{23}$. Além disso, a estratégia tática adotada pelas equipes 
também pode influenciar a maior exigência física imposta aos jogadores.

Com relação à distância percorrida nos três domínios fisiológicos (FIGURA 2) foi possível observar que os atletas permanecem maior parte do tempo se exercitando no domínio moderado quando comparado ao domínio pesado e severo, o que corresponde a praticamente 45\% da partida (FIGURA 3), considerado como um TE de "grande efeito", demonstrando assim uma predominância do metabolismo aeróbio.

O presente estudo também reportou que os atletas de F7 permanecem em média aproximadamente $33 \%(874,8 \mathrm{~m})$ do tempo total da partida executando açóes no domínio pesado, com valores de FC acima de $80 \%$ do PDFC e $23 \%$ (284,3 m) do tempo no domínio severo, com a FC acima do PDFC. Dessa forma, ao somarmos os valores de permanência nos domínios pesado e severo, podemos observar que mais de 50\% do tempo total de jogo foram situadas nestas intensidades, sendo que, do ponto de vista fisiológico, a permanência nestes dois domínios pode estar associada à melhora das adaptaçôes cardiovasculares e metabólicas ${ }^{24}$.

Estudos realizados com atletas de futsal demonstram que a maior parte do tempo os jogadores permanecem em atividades consideradas de intensidade elevada. No estudo de Arins e Silvi ${ }^{25}$, com treinamentos coletivos de futsal da categoria adulto, foi demonstrado que os atletas permaneceram a maior parte do tempo realizando açôes no domínio pesado. Do mesmo modo, Rodrigues et al. ${ }^{26}$ encontraram que em 13 partidas oficiais de futsal os atletas mantiveram a maior parte do tempo com valores de FC próximos a 90\% do máximo, que representa uma intensidade de esforço elevada. BARBERo-ÁllVAREZ et $\mathrm{al}^{4}{ }^{4}$, analisaram as demandas de 2 partidas de F7 em duas categorias (Sub 12 e 14) e demonstraram que ambas as categorias permaneceram em média $57 \%$ do tempo em intensidades acima de $85 \% \mathrm{FC}_{\text {MEDJOGO }}$. É importante destacar que devido à falta de estudos relacionados ao F7 na literatura científica com indivíduos adultos, torna-se difícil comparar e discutir os resultados do presente estudo. Entretanto, tal lacuna, justifica a importância da realização do presente estudo.

Por fim, por meio dos resultados obtidos neste estudo, podemos concluir que os atletas de F7 permaneceram a maior parte do tempo das quatro partidas amistosas realizando açóes com elevada demanda cardiovascular. Tais achados são de fundamental importância para os profissionais $\mathrm{da}$ área, uma vez que podem servir de referência para elaboração de programas de treinamento adequados às exigências fisiológicas da modalidade. Dessa forma, a partir dos resultados do presente estudo sugere-se a elaboração de sessóes de treinamento com predominância na melhora da capacidade aeróbia e como controle da carga de treinamento, a realização de atividades com intensidade acima de $80 \%$ do PDFC. Porém, vale ressaltar que este é o primeiro trabalho que analisou as demandas de jogos de F7 no Brasil e que, portanto, mais estudos são necessários para um melhor entendimento e conhecimento acerca dessa modalidade.

\section{Abstract}

\section{Intensity of effort during Football 7 exhibition matches}

The aim of the present study was to identify the intensity of effort demanded in 4 friendly matches and the distance covered in each of the physiological domains (moderate, heavy and severe). Ten adult players $(25.2 \pm 3.7$ years; $72.0 \pm 8.9 \mathrm{~kg} ; 175.3 \pm 6.9 \mathrm{~cm} ; 12.4 \% \pm 3.1$ body fat $)$ from an amateur 7 -a-side team took part of the study. Participants undertook five experimental sessions with an interval of at least 48 hours between each session. The first experimental session consisted of anthropometric measures followed by an intermittent field test (T-CAR) for the assessment of the maximal heart rate $\left(\mathrm{HR}_{\mathrm{Max}}\right)$, peak velocity $(\mathrm{PV})$ and for the identification of the physiological domains based on the heart rate deflection point. In the following sessions, the heart rate and the players activity profile (global positioning system - GPS) were measured during four friendly games. ANOVA two way for repeated measures was used in combination with post hoc testing Bonferroni. Statistically significant differences were not found in mean values of HR $(p<0.05)$ between games and the 1 st and 2 nd time. Related to the distance covered, the results showed that athletes spent most part of the time exercising in the moderate domain $(664.2 \pm 62 \mathrm{~m} ; 45.5 \%)$ when compared to heavy $(437 \pm 45 \mathrm{~m} m ; 31,5 \%)$ and the severe $(284.3 \pm 66 \mathrm{~m} ; 23.5 \%)(p<0.05)$. However, when we analyze the 
heavy and the severe domain together, it can be observed that $50 \%$ of the total game time was spent at high intensities. Thus, it can be concluded that the 7-a-side soccer is a high physiological demanding exercise.

KeYwords: Team Sports; Exercise Intensity; Heart Rate; Performance.

\section{Referências}

1. Confederação Brasileira de Futebol 7 [homepage na Internet]. [Localização desconhecida]: Portal 4 Linhas; [citado em 2018 mar 12]. Disponível em: <https://goo.gl/s34eNA>.

2. Nunes RFH, Almeida FAM, Santos BV, Almeida FDM, Nogas G, Elsandegy HM, et al. Comparação de indicadores físicos e fisiológicos entre atletas profissionais de futsal e futebol. Motriz. 2012;18(1):104-12.

3. Barbero-Álvarez JC, Soto VM, Barbero-Álvarez V, Granda-Vera J. Match analysis and heart rate of futsal players during competition. J Sports Sci. 2008;26(1):63-73.

4. Barbero-Álvarez JC, Gómez-López M, Castagna C, Barbero-Alvarez V, Romero DV, Blanchfield AW, et al. Game demands of 7-a-side soccer in young players. J Strength Cond Res. 2017;31(7):1771-9.

5. Gómez DC, Román-Quintana JS, Paulis JC, Calleja-González J. Demandas físicas y fisiológicas en jugadores absolutos no profesionales durante partidos de fútbol 7: un estudio de caso. CCD. 2012;7(20):115-23.

6. Lambert MI, Borresen J. Measuring training load in sports. Int J Sports Physiol Perform. 2010;5(3):406-11.

7. Nakamura FY, Moreira A, Aoki M.S. Monitoramento da carga de treinamento: a percepçáo subjetiva do esforço da sessão é um método confiável? Rev Educ Fís/UEM. 2010;21(1):1-11.

8. Coelho DB, Coelho LG, Mortimer LAF, Hudson ASR, Marins JCB, Soares DD, et al. Avaliação da demanda energética e frequência cardíaca em diferentes fases durante jogos ao longo de uma competição oficial de futebol. Rev Bras Cineantropom Desempenho Hum. 2012;14(4):419-27.

9. Helgerud J, Engen LC, Wisloff U, Hoff J. Aerobic endurance training improves soccer performance. Med Sci Sports Exerc. 2001;33(11):1925-31.

10. da Silva JF, Guglielmo LGA, Carminatti LJ, de Oliveira FR, Dittrich N, Paton CD. Validity and reliability of a new field test (Carminatti's test) for soccer players compared with laboratory-based measures. J Sports Sci. 2011;29(15):1621-8.

11. Almeida EB, Carminatti LJ, de Oliveira FR. Domínios fisiológicos na atividade ocupacional do carteiro ciclista. Rev Bras Fisio Exerc. 2004;3(1):119.

12. Conconi F, Borsetto C, Ziglio PG, Droghetti P, Codeca L. Determination of the anaerobic threshold by a noninvasive field test in runners. J Appl Physiol. 1982;52(4):869-73.

13. Dittrich N, da Silva J, Castagna C, de Lucas RD, Guglielmo LG. Validity of Carminatti's test to determine physiological indices of aerobic power and capacity in soccer and futsal players. J Strength Cond Res. 2011;25(11):3099-106.

14. Reid M, Duffield R, Dawson B, Baker J, Crespo M. Quantification of the physiological and performance characteristics of on-court tennis drills. Br J Sports Med. 2008;42(2):146-51.

15. Gil AC. Como elaborar projetos de pesquisa. $3^{\text {a }}$ ed. São Paulo: Atlas; 1991. p. 158.

16. Benedetti TRB, Pinho RA, Ramos VM. Dobras cutâneas. In: Petroski EL. Antropometria: técnicas e padronizaçóes. 2a ed. Porto Alegre: Pallotti; 2003.

17. Jackson AS, Pollock ML. Generalized equations for predicting body density of men. Br J Nutr. 1978;40(3):497-504.

18. Siri WE. Body composition from fluid space and density. In: Brozek J, Hanschel A, editores. Techniques for measuring body composition. Washington: National Academy of Science; 1961.

19. Tanaka H, Monahan KD, Seals DR. Age-predicted maximal heart rate revisited. J Am Coll Cardiol. 2001;37(1):153-6.

20. Kara M, GokbeL H, Bediz C, Ergene N, Uçok K, Uysal H. Determination of the heart rate deflection point by the Dmax method. J Sports Med Phys Fitness. 1996;36(1):31-4.

21. Cohen J. Statistical power analysis for the behavioral sciences. $2^{\mathrm{a}}$ ed. Hillsdale: Lawrence Erlbaum Associates; 1988.

22. Rahnama N, Reilly T, Lees A, Graham-Smith P. Muscle fatigue induced by exercise simulating the work rate of competitive soccer. J Sports Sci. 2003;21(11):933-42.

23. Mohr M, Krustrup P, Bangsbo J. Fatigue in soccer: a brief review. J Sports Sci. 2005;23(6):593-9.

24. Castagna C, Belardinelli R, Impellizzeri FM, Abt GA, Coutts AJ, D'Ottavio S. Cardiovascular responses during recreational 5-a-side indoor-soccer. J Sci Med Sport. 2007;10(2):89-95. 
25. Arins FB, Silva RCR. Intensidade de trabalho durante os treinamentos coletivos de futsal profissional: um estudo de caso. Rev Bras Fisio Exerc. 2007;9(3):291-6.

26. Rodrigues VM, Ramos GP, Mendes TT, Cabido CE, Melo ES, Condessa LA, et al. Intensity of official futsal matches. J Strength Cond Res. 2008;25(9):2482-7.

\begin{tabular}{|c|c|}
\hline ENDEREÇO & \\
\hline $\begin{array}{l}\text { Renan Felipe Hartmann Nunes } \\
\text { rtos da Universidade Federal de }\end{array}$ & Recebido para publicação: 16/o6/2016 \\
\hline $\begin{array}{r}\text { Santa Catarina } \\
\text { David Ferreira }\end{array}$ & Revisado: $28 / 07 / 2016$ \\
\hline Trindade & Aceito: 16/o8/2016 \\
\hline $\begin{array}{l}\text { - Florianópolis - SC - BRASIL } \\
\text { e-mail: reibinatti@hotmail.com }\end{array}$ & \\
\hline
\end{tabular}

